\title{
Active feedback control used in plasma vertical unstable displacement for EAST
}

\author{
Hai-hong HUANG, Ye WANG, Hai-xin WANG \\ Hefei University of Technology \\ Hefei 230009, China \\ Hhaihong741@126.COM
}

\begin{abstract}
EAST (Experimental Advanced Superconducting Tokamak), a National Mage-project of Science Research of China, is a very important fusion experiment facility. The ability of magnetic confinement to plasma can be improved by elongating plasma cross-section to form divertor configuration in EAST. But elongated plasma has vertical displacement instability, without control, plasma will dash against wall of vacuum vessel and disrupt, that will cause failure of plasma discharge. So feedback control system is needed to restrain plasma vertical displacement. Fast control power supply excites active feedback coils, which produces magnetic field to control plasma's displacement. The validity of proposed scheme was confirmed by experiments, the active feedback system can reach design demand.
\end{abstract}

Keywords- EAST; Plasma; Vertical unstable displacement; Active feedback; Fast control power supply

\section{INTRODUCTION}

The toroidal magnetic constraint Tokamak device is most hopeful to build commercial nuclear fusion power reactor. But Tokamak is a kind of low- $\beta$ device. $\beta$ is the ratio of plasma pressure and magnetic energy density, which represents the ability of magnetic field to constrain the plasma. Considering the economic benefit, the average value of $\beta$ should be about $10 \%$ to build a commercial reactor. Therefore, how to increase the value of $\beta$ has become a very important subject among the international study of nuclear fusion. The most popular method of increasing value of $\beta$ is elongating plasma cross-section to form divertor configuration, [1] but elongated plasma has vertical displacement instability.

Some kind of disturbance will cause vertical displacement of the elongated plasma. As a result, plasma will move at the vertical direction. Without control, plasma will dash against the wall of the vacuum vessel and disrupt, which cause failure of discharge. The high heat load stress and a lot of runaway electrons caused by the disruption may be serious or even destructive. [2] So a feed back system is needed to restrain plasma vertical displacement.

\section{ANALysis OF Plasma Vertical Instability}

EAST is one of national key science technical projects and the first fully Superconducting Tokamak device in the world. It is an axial symmetrical toroidal system, made up by transformers used to excite current, toroidal field coils used to produce toroidal magnetic field, balance magnetic field coils used to control plasma's balance position and a vacuum vessel. Current in center solenoids produces changing magnetic field, which also produces an electric field, and these finally realize breakdown and produce plasma. Toroidal magnetic field and poloidal magnetic field make up a spiral magnetic field which make the current of plasma move along the spiral magnetic line so that plasma is constrained. Fourteen superconducting coils for poloidal magnetic field, PF1 PF14, locate at positions symmetrical about the equatorial plane, it is shown as Fig.1. [3]

For shape control of plasma, the vertical component of poloidal magnetic field is mainly used to control shape of plasma at $\mathrm{R}$ direction, while the radial component of poloidal magnetic field control shape of plasma at $\mathrm{Z}$ direction. Different radial magnetic field will change the shape of plasma. Interactions of plasma current and radial magnetic field caused by external coils will produce Lorentz force for the plasma at vertical direction.

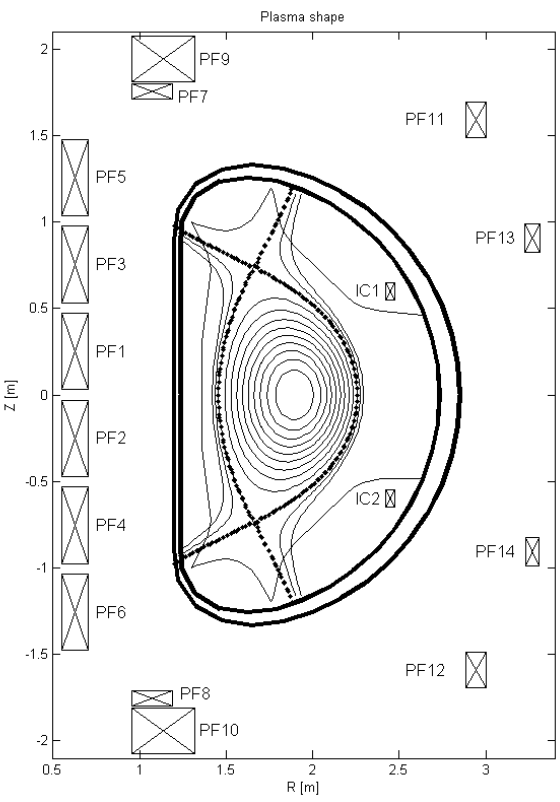

Fig.1 Position of EAST poloidal field coils

Define magnetic field reduction factor as $n$ (curvature of poloidal magnetic field):

$$
n=-\frac{R}{B_{\mathrm{Z}}} \frac{\partial B_{\mathrm{Z}}}{\partial R}
$$

$B_{\mathrm{Z}}$ is the component at $Z$ direction of extern poloidal magnetic field $B_{\text {ext. }}$. The force at $R$ direction from the component of extern magnetic field is:

$$
F_{\mathrm{Z}}=-2 \pi R_{0} B_{\mathrm{R}} I_{\mathrm{P}}
$$

$R_{0}$ is $R$ coordinate of the plasma current density centre. $I_{\mathrm{P}}$ 
is plasma current. $B_{\mathrm{R}}$ is the component at $R$ direction of extern poloidal magnetic field $B_{\text {ext }}$. According to Ampere rule: $\nabla \times B=\mu_{0} j$, considering the static magnetic field situation $(j=0)$, equation (3) can be obtained:

$$
\frac{\partial B_{\mathrm{Z}}}{\partial R}-\frac{\partial B_{\mathrm{R}}}{\partial Z}=0
$$

According to equation (1) (3), the force $F$ on plasma at vertical direction changes with the position of the plasma $Z$.

$$
\frac{\partial F_{\mathrm{Z}}}{\partial Z}=2 \pi R_{0} I_{\mathrm{p}} \frac{n B_{\mathrm{Z}}}{R}
$$

Fig. 2 shows two kinds of shape of plasma when it is elongated or flattened. It is shown that the shape of plasma is flat with a positive curvature, while the shape is elongated with a negative curvature. The curvature is zero when the magnetic field component at radial direction is zero, namely, the poloidal magnetic field don't change with $\mathrm{R}$ and $\mathrm{Z}$.
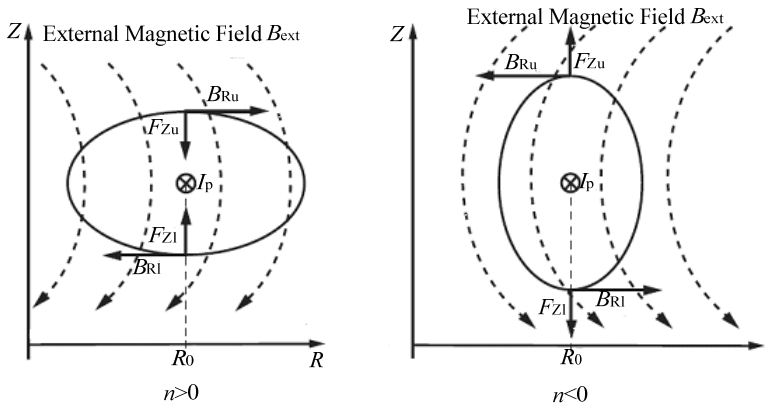

Shape of plasma with positive (b) Shape of plasma with negative curvature curvature

Fig.2 Plasma shape according to different $\mathrm{n}$ in axial magnetic field

When $B_{\mathrm{Z}}<0$, the balance situations of the plasma are as follows:

1) If $n>0, \frac{\partial F_{\mathrm{Z}}}{\partial Z}<0$, plasma is stable at vertical direction.

2) If $n<0, \frac{\partial F_{Z}}{\partial Z}>0$, plasma is unstable at vertical direction.

3) If $n=0, \frac{\partial F_{Z}}{\partial Z}=0$, plasma is at a critical state at vertical direction.

When plasma is elongated, it should be $n<0$. Therefore plasma is not stable at vertical direction, so a feedback control is needed.

\section{FEEDBACK CONTROL OF PLASMA VERTICAL DISPLACEMENT}

There are two ways of feedback control for plasma vertical displacement, passive feedback and active feedback. Passive feedback is a kind of fast response on millisecond level. Once plasma moves at vertical direction, conductor around the plasma (such as the metal wall of vacuum vessel) will induce eddy current, which will produce magnetic field. The radial component of the magnetic field BR interacting with the plasma current produces Lorentz force at opposite direction to plasma, so that the vertical displacement is suppressed. The passive feedback control of plasma in EAST is formed by double layer stainless steel vacuum vessel and two pairs of passive conductor plate. But passive feedback will be weakening after the conductor diffusion time because the eddy current will be dissipated by conductor resistance. Therefore, active feedback is needed for slow disturbance beyond the diffusion time scale. Method of active feedback is to sample signal of plasma's vertical displacement and feed it back to the control power supply, then current in the active feedback coils will be built to produce magnetic field to restrain the plasma's vertical displacement. The movement equation of plasma at vertical direction is:

$$
m_{\mathrm{P}} \frac{\mathrm{d}^{2} Z}{\mathrm{~d} t^{2}}=-2 \pi R_{\mathrm{p}} I_{\mathrm{p}} B_{\mathrm{R}}
$$

$m_{\mathrm{p}}, Z$ and $R_{\mathrm{P}}$ are respectively mass of plasma, vertical displacement and radius of plasma ring. To control plasma's vertical displacement, the increasing rate of plasma's vertical displacement $\gamma$ should be considered.

$$
\gamma(t)=\frac{\mathrm{d} \ln |Z(t)|}{\mathrm{d} t}
$$

The maximum increasing rate for EAST is $1000 / \mathrm{s}$, and the reaction rate of the active feedback coil should be faster than the increasing rate of the vertical displacement. The vacuum vessel causes $24 \mathrm{~ms}$ and $11.5 \mathrm{~ms}$ shielding delay for vertical component and radial component of magnetic field caused by poloidal magnetic field coils respectively, so fast control coils are needed in the vacuum vessel, it is shown in Fig.1. IC1 and IC2 is a couple of symmetrical active feedback coils as a saddle shaped connection across the vertical conductor. The current flowing in coils can build radial magnetic field to form a feedback control for plasma vertical displacement instability. The working principle of EAST active feedback coils is shown as Fig.3. A negative feedback model is shown as Fig.4.

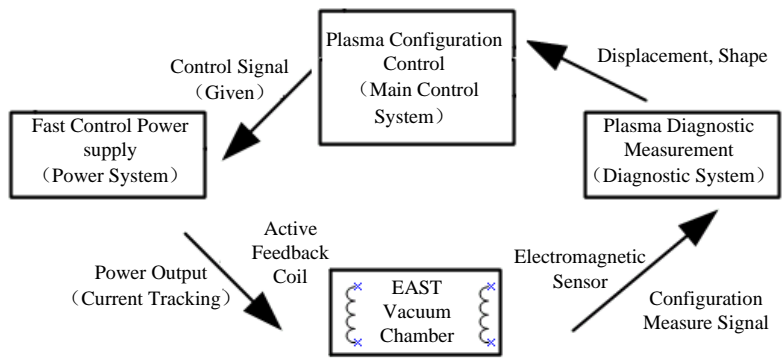

Fig.3 Principle of EAST active feedback coils

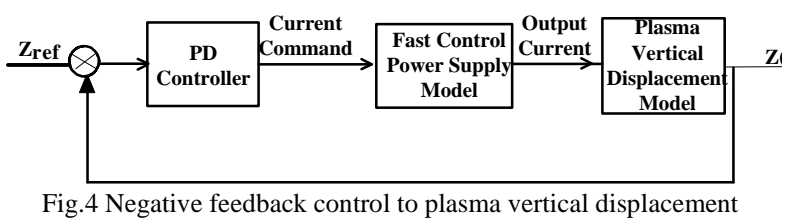

Plasma Control System (PCS for short) of EAST detects plasma vertical displacement, calculates given current signal for the main control power supply. The method of calculation is given by equation (7). The given current 
signal is linear amplified and real time tracked by the main control power supply.

$$
i_{\mathrm{c}}=-g_{\mathrm{P}} Z_{\mathrm{P}}+g_{\mathrm{d}} \frac{\mathrm{d} Z_{\mathrm{P}}}{\mathrm{d} t}
$$

$g_{\mathrm{P}}$ is proportion coefficient of controller, $g_{\mathrm{d}}$ is differential coefficient of controller, $Z_{\mathrm{P}}$ is plasma vertical displacement, $i_{\mathrm{c}}$ is needed output current of main control power supply. Plasma vertical displacement fast control power supply for
EAST builds up an active feedback control system of plasma vertical displacement with an active feedback coil in the vacuum vessel to realize the vertical balance and locating of plasma.

\section{FAST CONTROL POWER SUPPLY FOR ESAT}

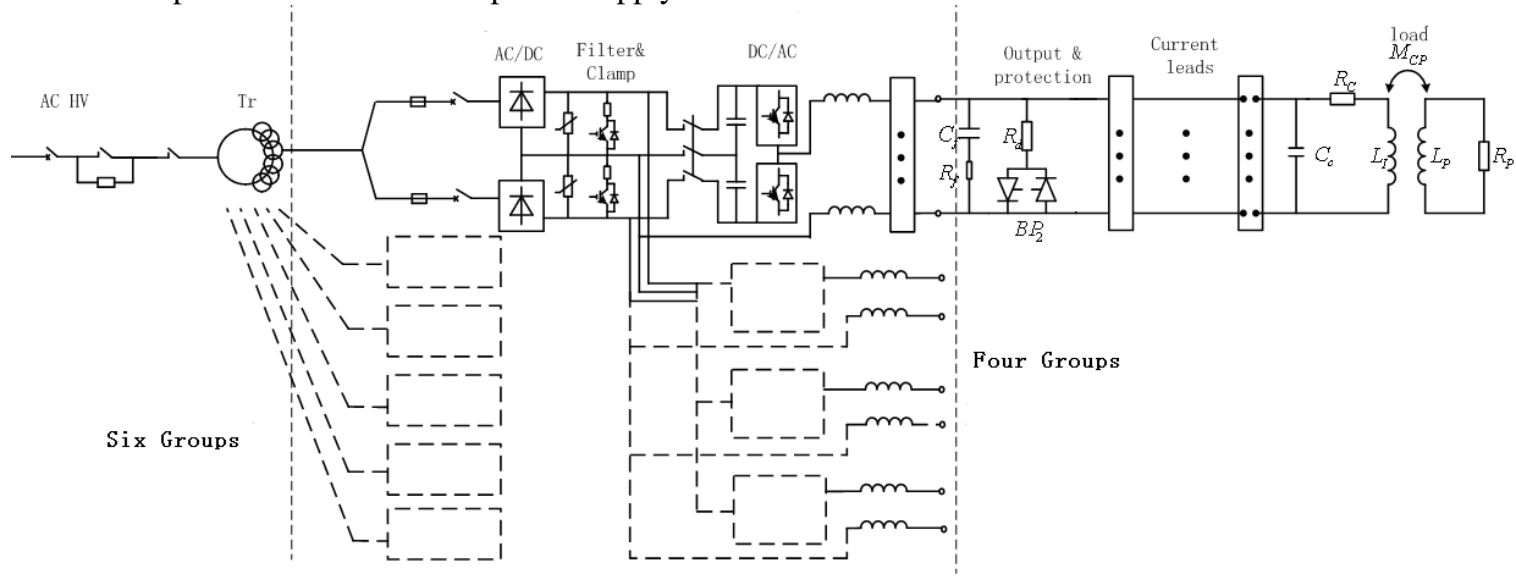

Fig.5 Structure of vertical displacement fast control power supply for EAST

The structure of vertical displacement fast control power supply for EAST is shown in Fig.5. [4] The transformer with 36 phases and uncontrolled rectifier with diodes are used as rectifiers in fast control power supply. The capacity of transformer is $560 \mathrm{kVA}$ and the voltage ratio of the primary side and the secondary side is $10 \mathrm{kV} / 0.7 \mathrm{kV}$. The phase differences between primary and secondary winding is respectively $\pm 5^{\circ}, \pm 15^{\circ}, \pm 25^{\circ}$. Two same winding of every phase are used as input power supply for a group of rectifier bridges. There are totally 6 groups of rectifier bridges. Every four invert units share one group of rectifier bridges. There is a sharing current reactor at the AC output side to limit the circulation current from parallel connection. [5]

\section{RESULT OF EXPERIMENT}

Fig.6 shows waves of plasma vertical displacement signal, PCS command, and current on internal coil. The tracking performance of fast control power supply is quite good and active feedback control can restrain the vertical displacement of the plasma well.

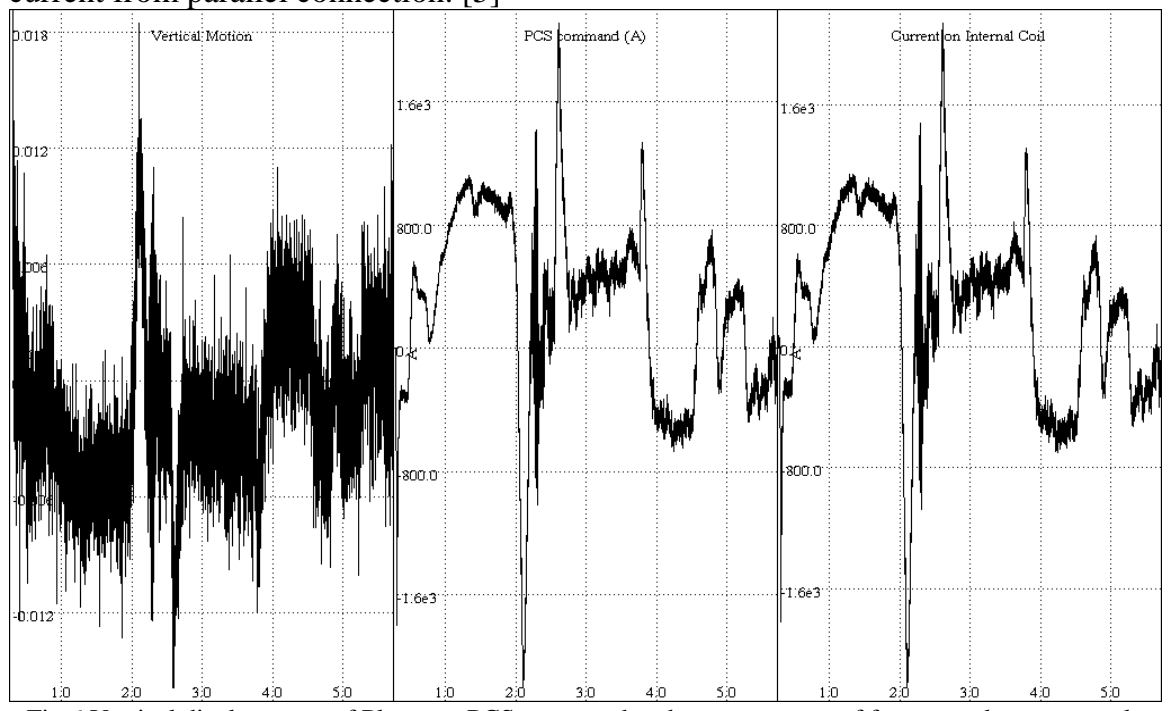

Fig.6 Vertical displacement of Plasma、PCS command and output current of fast control power supply 


\section{CONCLUSION}

Energy is the basis of human existence and society development. The research of nuclear fusion energy is an important way to solve the energy problems for mankind. Tokamak is hopeful to build fusion reactor. The method of form divertor configuration by elongating plasma in Tokamak device can improve the ability of magnetic field to constrain plasma. However, the vertical displacement of elongated plasma is not stable. The experiment results show that active feedback control can effectively prevent plasma vertical fast drift in large elongated shape, which is helpful to extend the discharge time.

Foundation: ITER Program of China (973 Program) (2011GB109002)

National Natural Science Foundation of China (11275056)

Hefei University of Technology Doctor Scientific Research Foundation (2011HGBZ1292)

\section{REFERENCE}

[1] Bondeson A, Liu D.-H., Söldner F.X, Persson M., Baranov Yu.F, Huysmans G.T.A. "MHD beta limits for advanced scenarios on JET" Nuclear Fusion. vol. 39, pp. 1523-1533, 1999.

[2] LIU Cheng-yue, CHEN Mei-xia, WU Bin. "Study on Vertical Displacement Instability on EAST Tokamak". Nuclear Fusion and Plasma Physics. vol. 29, pp. 301-304, 2009.

[3] LIU Cheng-yue, CHEN Mei-xia, SONG Feng-quan. "Numerical simulation of plasma current and configuration evolution in the EAST". Journal of Hefei University of Technology (Natural Science). vol.31, pp.314-316, 2008.

[4] Haihong Huang, Haixin Wang, Ge Gao, Peng Fu. “Application of Phase-shift PWM in EAST Fast Control Power Supply”. IEEE Transactions on Applied Superconductivity.vol.20, pp.1671-1675, 2010.

[5] HUANG Hai-hong, GAO Ge, FU Peng1, WANG Hai-xin, YAN Shi-chao. "Application of Three-level Large Power Paralleling Inverter in EAST System”. Nuclear Fusion and Plasma Physics. vol. 28, pp. 358-361, 2008. 